EPJ Web of Conferences 37, 06003 (2012)

DOI: $10.1051 /$ epjconf/20123706003

(C) Owned by the authors, published by EDP Sciences, 2012

\title{
Kaon pair production in proton-proton collisions at COSY-ANKE
}

\author{
Qiujian Ye 1,2 ,a
}

1 Department of Physics and Triangle Universities Nuclear Laboratory, Duke University, Durham, NC 27708, USA

${ }^{2}$ Institut für Kernphysik and Jülich Centre for Hadron Physics, Forschungszentrum Jülich, D-52425 Jülich, Germany

\begin{abstract}
The near-threshold production of kaon-pairs has been investigated in protonproton collisions at Cooler Synchrotron (COSY)at Forschungszentrum Jülich. In the $p p \rightarrow$ $p p K^{+} K^{-}$reaction, the invariant mass distributions of $K^{-} p, K^{-} p p$, and $K^{+} K^{-}$indicate the presence of both $K^{-} p$ and $K^{+} K^{-}$final state interactions. Total cross sections for $p p \rightarrow p p \phi$ reaction have been determined at excess energies $\varepsilon$ of $18.5,34.5$, and $76 \mathrm{MeV}$. The differential spectra at an excess energy of $18.5 \mathrm{MeV}$ exhibit a clear $s$-wave dominance, while the high statics data at an excess energy of $76 \mathrm{MeV}$ shows that higher partial waves represent the majority of the $p p \rightarrow p p \phi$ total cross section, whose energy dependence would then seem to require some $s$-wave $\phi p$ enhancement near threshold.
\end{abstract}

\section{Introduction}

Extensive measurements of kaon-pair and $\phi$-meson production in proton-proton collisions have been performed at COSY facilities. The $p p \rightarrow p p K^{+} K^{-}$reaction has been studied by the COSY-11 and COSY-ANKE collaboration for excess energies ranging from 3 to $108 \mathrm{MeV}$ [1-4]. The study of KN system can provide valuable information about the scattering length of kaon-nucleon interaction, and potentially, about intermediate hyperon states such as $\Lambda(1405)$ [5]. In all the $p p \rightarrow p p K^{+} K^{-}$data the $K^{-} p$ ad $K^{-} p p$ invariant mass distributions display a strong preferences for lower invariant masses, which may reflect an attraction between the $K^{-}$and one or both of the protons. The effects can be described by assuming factorized pair-wise final state interactions (FSI) in the $K^{-} p p$ systems [4]. However, the uncertainty on the effective scattering length for $K^{-} p$ interaction was very large. High statistics data are required in order to study the $K^{-} p$ FSI and the interaction between two kaons in detail.

Another prominent feature of the $p p \rightarrow p p K^{+} K^{-}$data is the production of $\phi(1020)$ meson which shows up as a clear peak in the $K^{+} K^{-}$invariant mass distribution. The near threshold $\phi$ production could provide a clean way of investigating the production mechanism of the $\phi$ meson and possible violations of the Okubo-Zweig-Iizuka (OZI) rule [6]. Besides the data collected by DISTO at an excess energy of $83 \mathrm{MeV}$ [7], we have previously measured the $p p \rightarrow p p \phi$ reaction at excess energies of $18.5,34.5$ and $76 \mathrm{MeV}$ at COSY-ANKE [3]. The total cross section for the $\phi$ production as a function of excess energy can be described by a calculation of three-body phase modified by the effects of $p p$ FSI. The differential cross section at the lowest energy of the ANKE data shows a clear $\mathrm{S}$ wave dominance and strong $p p$ final state interaction. While the work of the DISTO collaboration shows the importance of higher partial waves, they did not see any indication of $p p$ FSI in the proton-proton momentum spectrum. The lower statistics ANKE data at the two higher energies were sufficient to

\footnotetext{
${ }^{a}$ e-mail: qy4@phy.duke.edu
} 
exact total cross sections but it was hard to draw firm conclusions from the differential spectra. High statistic data are required to understand the $\phi$ production mechanism.

\section{Kaon-pair and $\phi$ meson production}

We present more precise data for the $p p \rightarrow p p K^{+} K^{-}$reaction at a beam energy of $\mathrm{T}_{p}=2.83 \mathrm{GeV}$ $(\varepsilon=76 \mathrm{MeV})$ obtained using the COSY-ANKE spectrometer. The experimental data were divided into two samples, a $\phi$-rich region where $1.01 \mathrm{GeV} / c^{2}<\mathrm{IM}_{K^{+} K^{-}}<1.03 \mathrm{GeV} / c^{2}$ and a non- $\phi$ (the rest) region.

The main feature of the non- $\phi$ data is the strong distortion of both the $K^{-} p$ and $K^{-} p p$ invariant mass distributions. The effects are more explicit if one forms the ratios of the differential cross section in terms of the invariant masses, as shown in Fig. 1. If the $K^{+} p$ and $K^{-} p$ final state interactions were identical, then the ratios would be constant and equal to unity. However, both $R_{K p}$ and $R_{K p p}$ display a strong preferences for lower invariant masses. The results are well reproduced by a simple factorized ansatz:

$$
F=F_{p p}\left(q_{p p}\right) \times F_{K p}\left(q_{K p_{1}}\right) \times F_{K p}\left(q_{K p_{2}}\right)
$$

where $q_{p p}, q_{K p_{1}}$ and $q_{K p_{2}}$ are the magnitudes of the relative momenta in the $p p$ and the two $K^{-} p$ systems, respectively. The $p p$ FSI was calculated using Jost function [4].

It is surprising that the distortions produced by the constant effective scattering length, $a=(0+$ 1.5i) fm, used at $\varepsilon_{K K}=51 \mathrm{MeV}$ [4] still describes the data well even at an excess energy with respect to the $K^{+} K^{-}$threshold as high as $108 \mathrm{MeV}$. This parametrization also describes well the ANKE data at different energies and also the COSY-11 results in the vicinity of threshold. However, this simple ansatz underestimates the cross section for low $K^{+} K^{-}$masses, i.e., in the interval between the $K^{+} K^{-}$ and $K^{\circ} \bar{K}^{\circ}$ thresholds at 987.4 and $995.3 \mathrm{MeV} / c^{2}$. Although the enhancement can be due to $K^{+} K^{-}$final state interactions, a combined analysis of ANKE data suggests that the effect can be understood in terms of an elastic $K+K$ - rescattering plus a contribution coming from the production of a $K^{\circ} \bar{K}^{\circ}$ pair followed by a charge-exchange rescattering. The inclusion of these contributions led to a better description of the $K^{+} K^{-}$invariant mass distribution and the energy dependence of the total cross section with respect to the excess energy. High statistics data have been collected at an excess energy of $25 \mathrm{MeV}$ for further investigations. The data are currently under analysis.

Having a good understanding of the background under the $\phi$ peak, it is possible to extract the invariant mass and angular distributions for the $p p \rightarrow p p \phi$ reaction. A truncated partial wave decomposition of the $p p \phi$ reaction has been proposed to fit the experimental data. The model includes only typical partial waves, where the spin-averaged squard transition matrix element is written as [8]:

$$
\begin{aligned}
\overline{|M|^{2}}= & A_{S s}(\hat{k} \times \hat{K})^{2}+A_{P S} \mathbf{p}^{2}+A_{P p}(\mathbf{q} \cdot \mathbf{p})^{2} \\
& +A_{S p}\left[3(\mathbf{q} \cdot \hat{K})^{2}-\mathbf{q}^{2}\right] .
\end{aligned}
$$

The momenta of the beam proton and the $\phi$ meson in the overall c.m. system are denoted by $\mathbf{K}$ and $\mathbf{q}$, respectively, $\mathbf{k}$ represents the momentum of decay kaons in the $\phi$ reference frame, and $\mathbf{p}$ is the relative momentum in the final $p p$ system. The $A_{S s}$ and $A_{S p}$ contributions were multiplied by an enhancement factor calculated by the Jost function which accounts for $p p$ FSI.

Monte Carlo simulations based on GEANT4, which took into account the detector efficiency, resolution and kaon decay probability, have been applied to perform acceptance corrections. The luminosity used for the analysis was determined on the basis of the $p p$ elastic scattering data. The acceptance

corrected angular distributions for the $p p \rightarrow p p \phi$ reaction measured in this experiment and that of DISTO are shown in Fig. 2. Systematic uncertainties arise from the background subtraction, tracking efficiency, and the model dependent acceptance corrections. In the near-threshold region where the $S s$ final state dominates the $\phi$ meson spin must lie along the beam direction. The polar angular distribution of the decay kaons in the $\phi$ meson rest frame should then display a $\sin ^{2} \theta_{\phi}^{K}$ distribution, where $\theta_{\phi}^{K}$ is the angle of a daughter kaon from the $\phi$ decay in the $\phi$ rest frame. Our measurement shown in Fig. 2a is consistent with DISTO result within the statistical uncertainties, which shows that the higher 

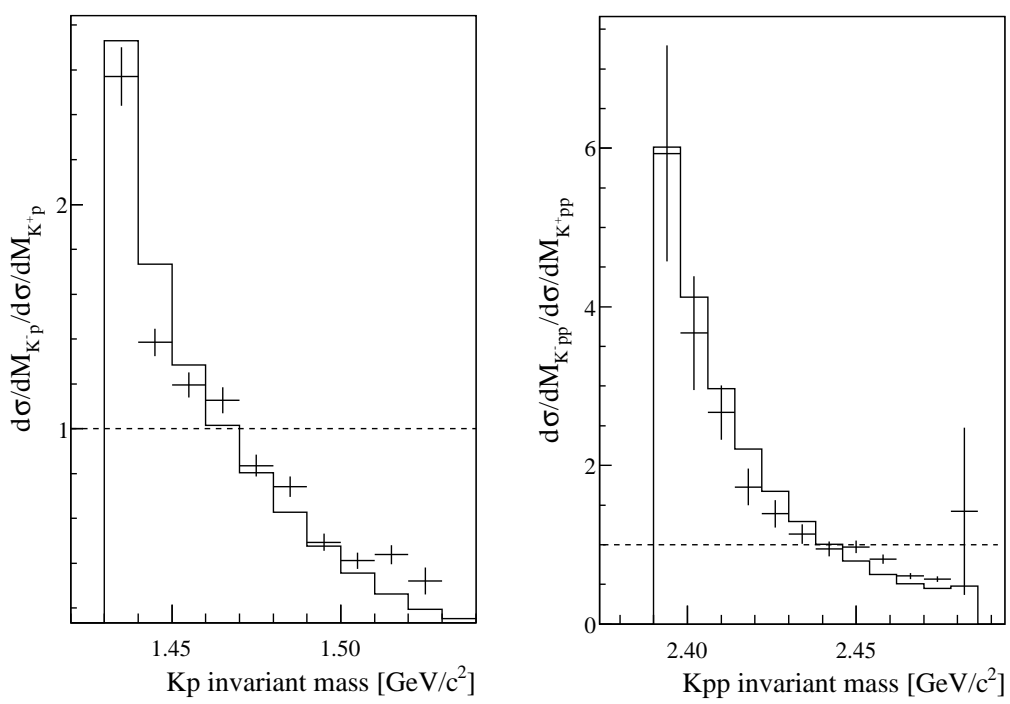

Fig. 1. Ratio of the differential cross section for the $p p \rightarrow p p K^{+} K^{-}$reaction in terms of the $K^{ \pm} p$ (left) and $K^{ \pm} p p$ (right) invariant masses. The dashed lines represent the four-body phase-space simulations, whereas the solid ones represent the theoretical calculations taking into account the $p p$ and $K^{-} p$ final state interactions.

partial waves are important at even relatively modest excess energies. The angular distribution of the $\phi$ meson in the overall c.m. frame shown in Fig. $2 b$ is symmetric within experimental uncertainties. The angular distribution is expected to be isotropic when the mesonic current is dominant, whereas the nucleonic current leads to a $\cos ^{2} \theta$ distribution. The angular distribution might therefore provide some information on the $N N \phi$ coupling constant $[9,10]$. The distribution in the proton polar angle measured in the $p p$ reference frame relative to the beam direction is nearly isotropic, as shown in Fig. 2c. On the other hand, the analogous observable relative to the $\phi$ direction shown in Fig. $2 \mathrm{~d}$ has some anisotropy. This feature, which was also seen in the DISTO data [7], is evidence for a contribution from a $P p$ final wave. The dominance of $\mathrm{P}$ waves in the final $p p$ system can explain why the ${ }^{1} S_{0} p p$ FSI, which is so important at $\varepsilon_{\phi}=18.5 \mathrm{MeV}$ [3], is not observed at $76 \mathrm{MeV}$.

If the coefficients $A_{L \ell}$ were constant, then they could be used to predict the energy dependence of the total cross section. However, the truncated model underestimates the total cross section at low energies, which might be due to the energy dependence of the fitting parameters. The simplest way out of the total cross section dilemma would be to assume a $\phi-p$ near threshold enhancement. Nevertheless, the phenomenological parametrization is sufficient for acceptance correction and it describes most of the differential distributions quite well.

Although some theoretical models have been able to describe the total cross sections for $\phi$ production, calculations of differential distributions are rather limited to be compared with our experimental data. Describing total cross sections is insufficient and more theoretical work is therefore required.

\section{Acknowledgements}

I would like to thank the COSY machine crew for their continued assistance as well as that of other members of the ANKE Collaboration. This work has been supported in part by the US Department of Energy under Contract No. DE-FG02-03ER41231, the BMBF, DFG, Russian Academy of Sciences, and COSY FFE. 


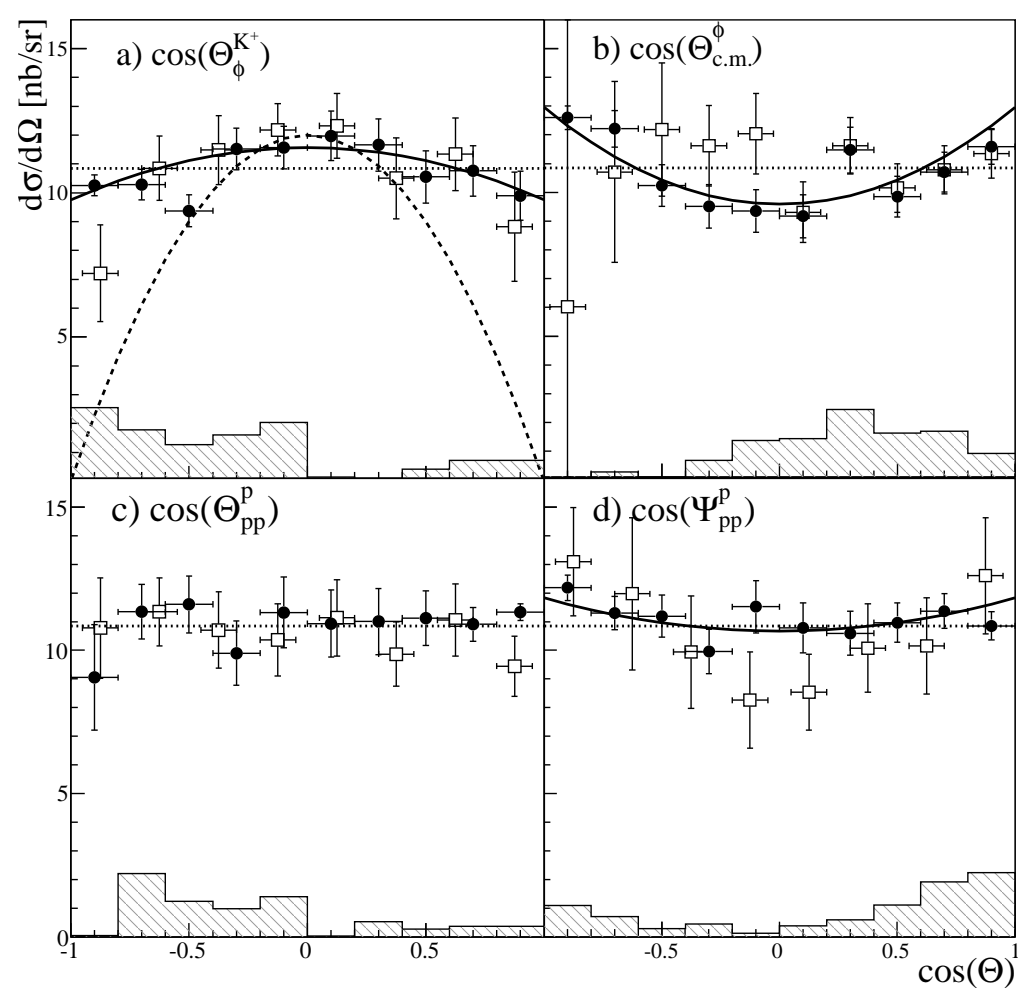

Fig. 2. Angular distributions of the $p p \rightarrow p p \phi$ reaction obtained in this experiment (solid circles), where the systematic uncertainties are shown by the hatched histograms, compared with the scaled DISTO data (open squares) [7]. The dotted curves represent isotropic distributions whereas the solid ones show fits to the ANKE results. (a) The distribution with respect to the cosine of the $K^{+}$polar angle in the $\phi$ rest frame (Decay angle). The dashed curve demonstrates a $\sin ^{2} \theta_{\phi}^{K}$ behavior. (b) The distribution in the $\phi$ polar angle in the overall c.m. system. (c) The distribution of the proton polar angle in the $p p$ reference frame relative to the incident proton direction (Jackson angle). (d) The distribution of the proton polar angle in the $p p$ reference frame relative to the $\phi$ direction (Helicity angle).

\section{References}

1. C. Quentmeier et al., Phys. Lett. B 515, 276 (2001).

2. P. Winter et al., Phys. Lett. B 635, 23 (2006).

3. M. Hartmann et al., Phys. Rev. Lett. 96, 242301 (2006).

4. Y. Maeda et al., Phys. Rev. C 77, 015204 (2008).

5. C. Wilkin, Acta Phys. Polon. Suppl. 2, 89 (2009).

6. S. Okubo, Phys. Lett. 5, 165 (1963); G. Zweig, CERN Report No. TH-401, 1964; J. Iizuka, Prog. Theor. Phys. Suppl. 37-38, 21 (1966).

7. F. Balestra et al., Phys. Rev. C 63, 024004 (2001).

8. Q. J. Ye et al., Phys. Rev. C 85, 035211 (2012).

9. K. Nakayama et al., Phys. Rev. C 60, 055209 (1999).

10. K. Tsushima and K. Nakayama, Phys. Rev. C 68, 034612 (2003). 